\title{
A modified mathematical model for lifetime enhancement in wireless sensor network
}

\author{
Sonali Banerjee, Ananya Ghosh, Swarup K. Mitra \\ Department of Electronics and Communication Engineering, M.C.K.V. Institute of Engineering, Liluah, \\ Howrah, 711204, India \\ Email: sonalibj8@gmail.com
}

\begin{abstract}
Enhancement of lifetime in static sensor network is an exigent task owing to limited power supply and restricted accessibility of sensor nodes. At every round of data transfer not all source nodes necessitate sending data to sink and hence tune itself to sleep mode. This paper utilizes the sleep-mode nodes as relays in transferring data from a transmitter to receiver for boosting energy efficiency in the network. Simple geometrical properties in modeling are used in the mathematics involved for choosing the most practicable sleep-mode node to relay the data. Moreover, such an approach requires the knowledge of geographical location of only the nodes in-between the transmitter and receiver. The simulation model use Poisson distribution to determine the sleep-mode nodes. This model is imposed on a range of data routing and clustering algorithm present in literature and results indicated a significant improvement in lifetime using it
\end{abstract}

Keywords: Relay Nodes, Sleep-mode Nodes, Network Lifetime, Lbeera, Pegasis, Short, HDS, Mathematical Models.

\section{INTRODUCTION}

Advancement in technology made the manufacturing of small and low-cost sensors technically and economically feasible. Those sensors are composed of a single chip with embedded memory, processor, and transceiver. Such sensors are also equipped with data processing and communication capabilities generally. Recent advances in micro-electromechanical systems and low power - highly integrated digital electronics have led to the development of micro-sensors [2]; [3], [4]. The sensing electronics of the sensors measure ambient condition related to the environment surrounding the sensor and then it transforms them into an electric signal. Processing such a signal reveals important properties about objects located and/or events happening in the vicinity of that particular sensor. A large number of these disposable sensors can be grouped or networked in many applications that require unattended operations. Wireless Sensor network consists of these sensor nodes densely or sparsely deployed in remote areas. The key feature of a typical sensor network is that their nodes are un-tethered and unattended. These sensors possess the ability to communicate either among each other or directly to an external base-station (BS). No doubt a greater number of sensors allows for sensing over larger geographical regions with greater accuracy than small number of sensors. Wireless sensor Networks may be deployed in inaccessible and hostile environments like dense jungles (for habitat monitoring applications), battlefields (for enemy troop movement monitoring), under the river (for flood monitoring) etc. These environments are not amenable to careful deployment of sensor nodes. So, it is expected that a large number of cheap, simple sensor devices will be randomly scattered over the region of interest. These devices are then expected to form a multi-hop network and relay critical information back to a "sink" which acts as a gateway to a backbone network. Thus, every node bears the burden of relaying information from other sensors in the network to the sink in addition to the sensing task. A network after deployed with large number of sensor nodes remains functional [5] only when all nodes or a predefined percentage of nodes are alive in that Network. During data gathering process between nodes to base station all the nodes don't actively involve in transmission and reception of data simultaneously. Therefore, necessity of utilizing the inactive nodes for a particular round of transmission as relay improves the network performance. Network lifetime [6] thus becomes an important parameter for efficient design of sensor networks. In case of WSNs, the lifetime can be taken as the time from inception of the nodes to the time when the network becomes non-functional. A network may become non-functional when a single node dies or when a particular percentage of nodes die depending on requirement. Each node is provided with transmit power control and Omni directional antenna and therefore can vary the areas of its coverage [7]. Each node is battery powered and has limited processing and memory capabilities. Therefore, it is critical that these resources are to be used in a judicious manner in order to maximize the benefit from the network before it dies. Although there is a cooperative effort 
from the device research community towards designing low power hardware and efficient energy sources, the network research community has also realized that inefficient algorithms at the various networking layers can result in nodes dying prematurely. There are several proposals at the MAC [8].

Routing in WSNs is very challenging due to its inherent characteristics that distinguish these networks from other wireless networks like mobile ad hoc networks or cellular networks. Firstly, as the number of sensor nodes is relatively high, it is not feasible to build a global addressing scheme for the deployment of a large number of sensor nodes as the overhead of ID maintenance is high. Secondly, almost all applications of sensor networks require the flow of sensed data from multiple sources nodes to a particular BS, in contrast to typical communication networks. Thirdly, as the sensor nodes are tightly constrained in terms of energy, processing, and storage capacities, they require careful resource management. Fourthly, in most application scenarios, nodes in WSNs are generally stationary after deployment except for, may be, a few mobile nodes. Fifthly, position awareness of all sensor nodes is important since data collection is normally based on the location. There are various routing techniques present in many articles A details discussion of the available routing algorithms is presented in the next section.

In this paper, we will introduce a new routing technique to enhance the performance of the network. This technique if mainly based on the consideration of sleep nodes (the inactive nodes of a particular time interval) as relay nodes. In the context of number of nodes to be acting as relay depends on the communication range [9] and application of the network. This analysis is emphasized by a distribution function over node deployment in a sensor network. The selection of relay nodes is based on Poisson distribution function in accordance with the application of the sensor network. Numerous energy efficient protocols have been proposed to route data to the BS. Many of them take a cluster-based approach [10]); a few sensor nodes are elected as cluster-heads (CHs) to collect data from their cluster members. The data traffic can be greatly reduced by applying data aggregation at CHs. Cluster members have low energy consumption, as they transmit their data to its nearby $\mathrm{CH}$. For better load balance, the role of $\mathrm{CH}$ is rotated among cluster members. Lindsey et al. presented several chain-based protocols for sensor network. They adopted a chain-based approach for situation awareness systems and proposed a linear-chain scheme for all-to-all broadcasting and data gathering. For data gathering and fusion, each node senses and transfers data along the chain to reach one particular node called leader that will send data to the distant BS. Such a scheme is named as PEGASIS [6]. In this paper, we propose a novel energy aware relay routing of data, controlled by BS, to reduce energy consumption and construct the lower chain in each cluster. A key factor of energy consumption for data communication is the transmission distance. The proposed algorithm is effective to avoid the formation of Long Link (LL) between neighboring nodes and therefore outperforms the existing chain construction algorithm protocols. Also, LBEERA [11] selects leader for each lower chain according to the remaining energy of nodes and chooses super leader among all leaders for higher chain according to the residual energy of leader and the distance between leader and the BS. In order to reduce the overhead of re-elected leader and super leader, we prefer to select leader and super leader every several rounds rather than each round [12].
The rest of this paper is organized as follows. Section 2 deals with related works. Section 3 the proposed algorithm together with an in-depth analysis of some important performance metrics. Simulation results for different routing schemes and network sizes are compared and discussed in Section 4, followed by conclusions in Section 5.

\section{RELATED WORKS}

In this section, we will discuss the routing protocols present for WSNs. In general, routing in WSNs is divided into three major groups based on network structure- flatbased routing, hierarchical-based routing, and location-based routing. Now all nodes are typically assigned equal roles or functionality in case of flat based routing. SPIN [13] are two examples of flat based routing techniques. All the nodes will play different roles in the network in case of Hierarchicalbased routing e.g. LEACH [10], PEGASIS [14]. In locationbased routing like GAF, sensor nodes' positions are used to route data in the network. Furthermore, based on the protocol operation these protocols can also be classified into Multipath-based, Query-based, Negotiation-based, QoSbased, and Coherent-based routing techniques. Multipathbased routing uses multiple paths rather than a single path in order to enhance the network performance. In case of Query based routing the destination nodes floods a query for data from a node through the entire network and a node having this data sends the data which matches the query back to the node, which initiates the query. While negotiation-based routing uses high level data descriptors in order to eliminate redundant data transmissions through negotiation, QoS-based routing protocols focuses towards maintaining balance between energy consumption and data quality. In addition to the above, routing protocols can again be classified depending on how the source finds a route to the destination into three categories, namely, Proactive, Reactive, and Hybrid protocols. In case of Proactive or Table-driven routing, up-to-date routing information is maintained and routing overhead is independent of route usage. In case of Reactive or Source initiated routing, explicit route discovery mechanisms are used and routes are maintained only for routes in use. Hybrid protocol is a combination of proactive and reactive routing methods.

The first flat routing protocol is proposed by Heinzelman, Kulik and Balakrishnan (1999) and Kulik, Heinzelman and Balakrishnan (2002). They proposed a family of adaptive protocols called Sensor Protocols for Information via Negotiation (SPIN) that broadcast all the information at each node to every node in the network assuming that all nodes in the network are potential base-stations. The SPIN family of protocols includes many protocols. The main two protocols are called SPIN-1 and SPIN-2. Other protocols of the SPIN family are SPIN-BC, SPIN-PP, SPIN-EC, and SPIN-RL. Intanagonwiwat, Govindan and Estrin, (2000) proposed a popular data aggregation paradigm for WSNs, called directed diffusion, which is a data-centric and application-aware concept in the sense that all data generated by sensor nodes is named by attribute-value pairs. The main concept of the datacentric theory is to coalesce the data coming from different sources enroute (in-network aggregation) by eliminating redundancy, minimizing the number of transmissions; Rumor routing is a variation of directed diffusion and is mainly intended for applications where geographic routing is not viable. The MCFA is another routing algorithm that exploits the fact that the direction of routing is always known, that is, 
towards the fixed external base-station. Schurgers, and Srivastava (2001) proposed another variant of directed diffusion, termed Gradient-Based Routing (GBR).

Table 1. Power outputs for the discrete power levels available

\begin{tabular}{|c|c|c|c|c|c|}
\hline $\begin{array}{c}\text { Power } \\
\text { Level }\end{array}$ & $\begin{array}{c}\text { Power } \\
\text { Output }\end{array}$ & $\begin{array}{c}\text { Power } \\
\text { Level }\end{array}$ & $\begin{array}{c}\text { Power } \\
\text { Output }\end{array}$ & $\begin{array}{c}\text { Power } \\
\text { Level }\end{array}$ & $\begin{array}{c}\text { Power } \\
\text { Output }\end{array}$ \\
\hline 0 & -37.90 & 11 & -10.00 & 22 & -3.520 \\
\hline 1 & -33.00 & 12 & -9.120 & 23 & -3.000 \\
\hline 2 & -28.70 & 13 & -8.330 & 24 & -2.470 \\
\hline 3 & -25.00 & 14 & -7.630 & 25 & -1.950 \\
\hline 4 & -21.80 & 15 & -7.000 & 26 & -1.450 \\
\hline 5 & -19.20 & 16 & -6.440 & 27 & -1.000 \\
\hline 6 & -16.90 & 17 & -5.940 & 28 & -0.610 \\
\hline 7 & -15.00 & 18 & -5.470 & 29 & -0.310 \\
\hline 8 & -13.40 & 19 & -5.000 & 30 & -0.091 \\
\hline 9 & -12.10 & 20 & -4.520 & 31 & 0 \\
\hline 10 & -11.00 & 21 & -4.030 & - & - \\
\hline
\end{tabular}

Heinzelman, Chandrakasan and Balakrishnan (2000) introduced the first hierarchical clustering algorithm for sensor networks, called Low Energy Adaptive Clustering Hierarchy $(\mathrm{LEACH})$. LEACH is a cluster-based protocol that includes distributed cluster formation. An enhancement over LEACH protocol was proposed in (Lindsey and Raghavendra, 2002). This new protocol, called Power-Efficient Gathering in Sensor Information Systems (PEGASIS)[6], is a near optimal chain-based protocol. The protocol is based on the basic idea that in order to extend network lifetime, nodes need only communicate with their closest neighbors and they take turns in communicating with the base-station. Another two hierarchical routing protocols called TEEN [15] (Threshold-sensitive Energy Efficient sensor Network protocol), and APTEEN (Adaptive Periodic Thresholdsensitive Energy Efficient sensor Network protocol) [16] are proposed in (Manjeshwar, and Agrawal, 2001; 2002), respectively. These protocols were proposed mainly for timecritical applications.

Two fundamental functions of the sensor nodes in a wireless sensor network are to sense its environment and to transmit sensed information to a base station. One approach to prolong sensor network lifetime is to deploy some relay nodes whose main function is to communicate with the sensor nodes, other relay nodes, and the base stations. It is desirable to deploy a minimum number of relay nodes to achieve certain connectivity requirement. In this paper, we study four related fault-tolerant relay node placement problems, each of which has been previously studied only in some restricted form.

\section{PROPOSED MATHEMATICAL MODEL AND BASIC CONSIDERATIONS}

We have segmented this section into three parts. In the first part, we have provided the power dissipation models used for experimental simulation and establishment for proving our model mathematically. In second part, we discussed the network level design (node deployment and the nature of the trans-receiving antenna), and in third part we provided the proposed mathematical model with algorithm

\subsection{The power dissipation models}

We have considered two different power dissipation models in this manuscript. For proving our model mathematically, the first order radio model is used and for experimental simulations the realistic power control model (Mitra et al., 2011) [17] is considered.

In first order radio model a radio dissipates energy and to transmit and receive a packet respectively. When a node acts as a relay the receiving energy, cost is way too low when compared to transmitting the same packet from that node as no network coding or data aggregation is performed. So here, only the mathematical equation for transmitting a packet of length ' $k$ ' over distance ' $d$ ' is considered as relevant and is given as

$$
E_{T x}(k, d)=e_{\text {amp }} * d^{\lambda} * k+E_{\text {elec }} * k
$$

\section{With $E_{T x}>E_{R x}$}

In equation (1) is the power dissipated to run the transmitter and has a value of $50 \mathrm{~nJ} / \mathrm{bit}$, is the energy dissipation by the transmission amplifier and is equal to $100 \mathrm{pJ} / \mathrm{bit} / \mathrm{m} 2$. represents the path loss exponent with. Here we have taken the path loss exponent as 2 to perform the distance calculation using Euclidian geometry that is the space dimension is an integer.

The realistic power model as described in [17] gives a realistic power consumption scenario. According to this model a sensor node works in 32 identifiable power levels with different power output for each level. This power level gets adjusted according to the distance of the node to which it wants to communicate.

The power levels of a CC2420 (C.T.Inc) (we have used the data sheet of this chip for our calculations) trans-receiver is given in table 1 . Out of this 32 power levels, (Mitra et al., 2011) has justified that only power levels $3,7,11,19,23,27$ and 31 are useful. The distance range in which a particular power level operates and the energy consumed for sending data packets in that power level is shown in table 2 . We have used the data of table 2 for calculating the energy consumptions and this gives a realistic power consumption model which can be used for simulation within, involving any hardware.

Table 2. Various power outputs for useful discrete power levels

\begin{tabular}{|c|c|c|c|c|c|}
\hline $\begin{array}{c}\text { Power } \\
\text { level } \\
(\mathbf{P})\end{array}$ & $\begin{array}{c}\mathbf{P}_{\text {out }} \\
{[\mathbf{d B m}]}\end{array}$ & $\begin{array}{c}\text { Distance } \\
(\mathbf{i n} \text { meters })\end{array}$ & $\mathbf{I}_{\mathbf{x}}(\mathbf{m A})$ & $\begin{array}{c}\mathbf{P}_{\mathbf{T x}} \\
(\mathbf{m W})\end{array}$ & $\begin{array}{c}\mathbf{E}_{\mathbf{t x}} / \mathbf{b i t} \\
{[\boldsymbol{\mu J}]}\end{array}$ \\
\hline 3 & -25.00 & $\mathrm{~d}<8.7 \mathrm{~m}$ & 17.04 & 15.15 & 0.0606 \\
\hline 7 & -15.00 & $8.7 \mathrm{~m}<\mathrm{d}<21.86$ & 15.78 & 17.47 & 0.0699 \\
\hline 11 & -10.00 & $21.86<\mathrm{d}<34.61$ & 14.63 & 19.62 & 0.0785 \\
\hline 19 & -5.00 & $34.61<\mathrm{d}<54.97$ & 12.27 & 22.08 & 0.0883 \\
\hline 23 & -3.00 & $54.97<\mathrm{d}<62.22$ & 10.91 & 26.33 & 0.1050 \\
\hline 27 & -1.00 & $62.22<\mathrm{d}<71.67$ & 9.71 & 28.40 & 0.1136 \\
\hline 31 & 0 & $74.47<\mathrm{d}<81$ & 8.42 & 30.67 & 0.1227 \\
\hline
\end{tabular}




\subsection{The network level design}

For assemblage of data, the wireless sensors motes are spatially distributed in a random topology. But there should be some pattern in the spatial distribution. Deployment of sensor nodes in topology is also a constraint on which energy efficiency of the network is restricted. Here we have incorporated an efficient node deployment strategy which improves the lifetime of the network (an experimental proof of this assertion is provided in the section 4 of this manuscript).

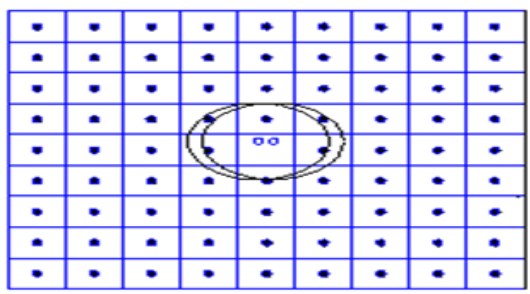

Figure 1. Representation of sensor deployment protocol to be adopted for enhancement of lifetime in wireless sensor network

We have used the realistic power control model in framing this protocol. Our intension is to make the motes to operate at the lowest possible power level considering the power level definitions as in [17]. There is no sense to place two source nodes in the range of their coverage as both of them would collect the same data. Optimization in the deployment of sensors in context of cost and energy dissipation is very important. For achieving this we divide the field in $\mathrm{n}$ squares of edge length ' $a / \sqrt{ } n$ ' for the deployment of ' $n$ ' sensor motes considering a square field of edge length ' $a$ '. This is shown in Figure 1. The nodes are deployed within each such sub squares on a randomly occupying position in those regions. For explanation, if we deployed two motes in one sub square then it can be seen that the coverage region of these nodes overlaps when each operates at the lowest possible power level. Thus, there is no need to place two source nodes within such close proximity or analogously in the same such square block. In other case when two or more source nodes are placed within the coverage region of one another it would be more power saving to switch the sensor motes off until the other gets exhausted in terms of power.

Now it can be also seen in Figure 1 (it's the most trivial deployment case considered here for elucidation) that by following this protocol each sensor has eight sensors surrounding its sensing region. Now data transfer also takes place at the lowest power level and hence the message transmission cost will also be low which enhances the lifetime of the network. As per the above theory a single source node can have 8 sensors to a minimum of 1 in its broadcast region when all the motes are operating at the same power level.

\subsection{The proposed mathematical model}

In this section, we introduce the mathematical model which efficiently judges an alternate path between a receiver and transmitter for data transfer. The alternate path must necessarily include a relay node which is in sleep mode and satisfy the mathematical inequality as stated in Lemma 1. Choosing any other node to act as a relay would cause data aggregation through network coding, resulting in excess power dissipation through the alternate path as the receiver energy dissipation would no longer be negligible when compared to transmitter dissipation (as elaborated in section 3.1). The sleep mode node would only relay the data without data processing.

Lemma1. During data transfer between two sensor nodes separated by a two-dimensional Euclidian distance ' $d$ ', the energy dissipated would be minimum if there exist another node at that instant of data transfer in sleep mode with twodimensional Euclidean distance from the two source nodes as ' $\mathrm{d}$ ' and ' $\mathrm{d}_{2}$ ' and satisfying the relation: $d_{1}^{2}+d_{2}{ }^{2}<d$ Proof:

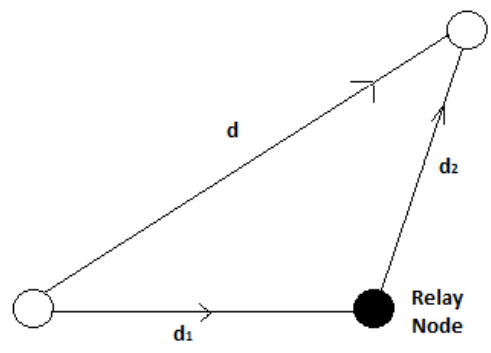

Figure 2. Routing between two nodes with a relay node (as sleep mode node)

As shown in Figure 2, let the Euclidean distance between two source nodes (indicated by white circles), between which data transfer takes place, be ' $d$ ' and the Euclidean distance between the transmitter and receiver node from the sleep mode node (indicated by black circle) be ' $\mathrm{d}_{1}$ ' and ' $\mathrm{d}_{2}$ ' respectively. So, by using the first order radio model we have the transmitted energy equation as

$E_{T x}(k, d)=e_{\text {anp }} * x^{2} * k+E_{\text {elec }} * k$

In general case the length of the packet is of order of 100 bits and if the sensor node uses CC2420 then it can operate as at a maximum distance of 90 . So the first term in eqn. 2 dominates over the second term and hence we can reframe the equation as

$E_{T x}(k, d)=e_{\text {anp }} * x^{2} * k$

Now, referring to Figure 2 we have the transmission energy dissipation for direct routing between the source nodes as

$E_{T 1 x}(k, d)=e d^{*} d^{2} * k$

and for routing between two nodes with sleep mode node as relay

$E_{T 2 x}(k, d)=e_{d} d_{1}^{2} * k+e d^{*} d_{2}^{2} * k$

For lesser power dissipation through the alternate path the inequality that must be fulfilled is

$E_{T 2 x^{(k, d)<}} E_{T 1 x}(k, d)$

Substituting the respective values of transmitted energy from equation 4 and 5 we have 
$e_{d}^{*} d_{1}^{2 * k+e_{d} * d_{2}^{2}{ }^{2} k<e_{d} * d}{ }^{2 * k}$

Or, $d_{1}^{2}+d_{2}^{2}<d^{2}$

Thus when the condition as stated in equation 8 is satisfied then by acting the sleep mode node as a relay node the energy consumption is greatly reduced. But, due to the approximation introduced in the calculations some error may be introduced and it must be taken into notice that this mathematical model is full proved only when the approximation is satisfied.

The above inequality is determined for all the nodes which can minimize the power dissipation in the network and the most excellent node in this respect is chosen. In order to improve the throughput of the network and to ensure that the transmitter node posses the geographical identity of minimum number of nodes to perform the computation according to above mathematical model, an efficient strategy must be employed. This strategy is produced in form of mathematical reasoning as stated in Lemma 2.

Lemma 2. The transmitter node needs to compute the equation 8 , to determine the best path of data transmission, for only a small fraction of all sleep-mode nodes. This small fraction must reside in the common region of the two circular regions with centers at source and sink respectively and radius equal to the Euclidian distance between the source transmitter node and receiver node.

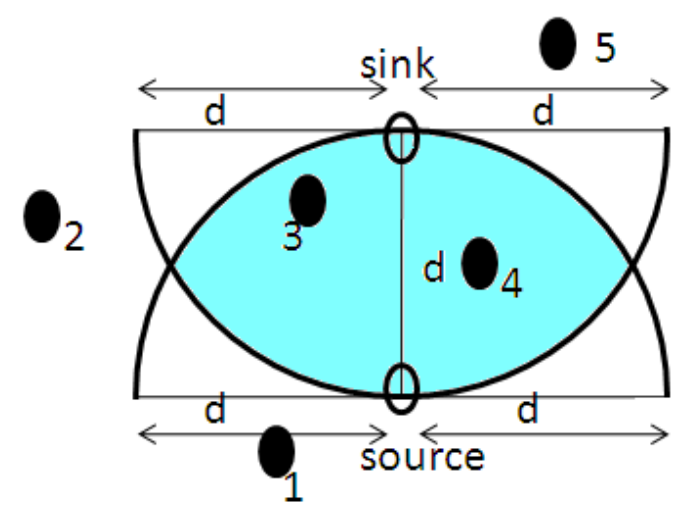

Figure 3. Figure showing the sleep mode nodes whose distances are to be calculated as they have the potentials to act as relay in data transfer

Proof: Let out of $\mathrm{n}$ nodes in a network at any particular round, ' $M$ ' nodes acts as a sleep node. Then instead of computing the distance $d_{1}$ and $d_{2}$ for each sleep nodes, it only computes the same for nodes within the sky blue region as shown in Figure 3.

Presume there are 5 nodes in sleep mode (as depicted in Figure 3 with sleep mode nodes marked as black ellipse and transmitting and receiving node as white ellipse) in the whole network and the transmitter (marked as source in Figure 3) needs to transmit its data to the receiver (marked as sink in Figure 3).

Outside the sky-blue region, the condition $\mathrm{d}_{1}^{2}+\mathrm{d}_{2}^{2}<\mathrm{d}^{2}$ is never satisfied as for any point outside the semicircle, one of the distance ' $\mathrm{d}_{1}$ ' or ' $\mathrm{d}_{2}$ ' has to be greater than $\mathrm{d}$ and hence the condition imposed by lemma 1 is never satisfied. So, it is impractical to perform the necessary computations for nodes outside that region for any transmitter receiver pair as it would add to a greater latency in the network.

Pseudo Code for Lemma 2

$\mathrm{d}=$ radius of the circles;

Distance (node (i), node (j)): Euclidian distance between i-th node and $\mathrm{j}$-th node (i.e. any two nodes in the network)

If distance (node (i), source) $<=\mathrm{d}$ and distance (node (i), sink) $<=\mathrm{d}$

$$
\begin{aligned}
& \text { If eqn. } 8 \text { is TRUE for node (i) } \\
& \text { It is the selected relay node; } \\
& \text { End }
\end{aligned}
$$

End

It should be noted that if none of the node qualifies as a relay then data transfer should proceed normally. The above model in whole is represented in a simple algorithmic form below for a particular transmitter receiver pair.

Input: Two communicating nodes with transmitter $=\mathrm{n}_{1}$ and receiver $=\mathrm{n}_{2}$

Output: The node which qualifies as relay or null

1. Draw a region as per Figure. 3 and determine the node identities of the sleep mode nodes and number of sleep mode nodes in that region and denote it by $\mathrm{m}$

2. If $m$ equal to null

2.1. Output: No node qualifies as relay, follow direct routing.

2.2. Else

2.2.1 For a variable $\mathrm{i}$ varying from 1 to $\mathrm{m}$ in steps of one

2.2.2. Compute distance between the $\mathrm{i}^{\text {th }}$ sleep mode node and the receiver and the transmitter respectively and store them in a array $d_{1}(i)$ and $d_{2}(i)$

2.2.3. Compute $\mathrm{d}_{3}(\mathrm{i})=\mathrm{d}_{1}{ }^{2}(\mathrm{i})+\mathrm{d}_{2}{ }^{2}(\mathrm{i})$

2.2.4. End for loop

2.3. Find the node having minimum magnitude $d_{3}$ in the array and label the node with variable $\mathrm{j}$

2.4 If $\mathrm{d}_{3}(\mathrm{j})<$ distance between $\mathrm{n}_{1}$ and $\mathrm{n}_{2}$

2.4.1. Output: Qualify node $\mathrm{j}$ as the relay node between the two nodes

2.4.2. Else

2.4.3. Output: No node qualifies as relay, follow direct routing

2.4.4. End if else statement

2.5. End if else statement

2.6. End program

The above algorithm is executed when any node in the network transmits a data packet to a different node.

\section{SIMULATION AND EXPERIMENTAL RESULTS}

In order to generate a real-time simulation, the nodes in a network that acts as sleep mode nodes are determined by Poisson Distribution Function i.e. by using the memory less property of Poisson Distribution Function the simulator identifies the inflow of critical information to a node which requires the data to be routed and in absence of critical information the node can act as sleep mode node thus saving energy in the network.

In probability theory and statistics, the Poisson distribution is a discrete probability distribution that expresses the probability of a number of events occurring in a fixed period of time if these events occur with a known average rate and independently of the time since the last event. If the expected 
number of occurrences in this interval is $\lambda$, then the probability that there are exactly $r$ occurrences $(r$ being a non-negative integer, $r=0,1,2 \ldots$. ) is equal to

$$
f(r, \lambda)=\frac{\lambda^{r} * e^{-\lambda}}{r !}
$$

Table 3. The network lifetime for various routing protocols with and without using relay concept with variation of parameter $\lambda$

\begin{tabular}{|c|c|c|c|c|c|}
\hline \multicolumn{3}{|c|}{\begin{tabular}{c} 
Value of the \\
parameter \\
\multicolumn{2}{|c|}{$\lambda$}
\end{tabular}} & \multicolumn{4}{|c|}{\begin{tabular}{c} 
Data Gathering Algorithm \\
\multicolumn{2}{|c|}{}
\end{tabular}} & \multicolumn{3}{|c|}{ (Netwom) square field of 100 sensors } \\
\cline { 3 - 6 } & SHORT & $\begin{array}{c}\text { LBEER } \\
\text { A }\end{array}$ & $\begin{array}{c}\text { PEGAS } \\
\text { IS }\end{array}$ & HDS \\
\hline 0.8 & $\begin{array}{c}\text { With } \\
\text { Relay }\end{array}$ & 1340 & 1190 & 900 & 1420 \\
\cline { 2 - 6 } & $\begin{array}{c}\text { Without } \\
\text { Relay }\end{array}$ & 1195 & 900 & 790 & 1010 \\
\hline 1.2 & $\begin{array}{c}\text { With } \\
\text { Relay }\end{array}$ & 1335 & 1110 & 860 & 1160 \\
\cline { 2 - 6 } & $\begin{array}{c}\text { Without } \\
\text { Relay }\end{array}$ & 910 & 990 & 580 & 890 \\
\hline 1.6 & $\begin{array}{c}\text { With } \\
\text { Relay }\end{array}$ & 900 & 840 & 715 & 920 \\
\cline { 2 - 6 } & $\begin{array}{c}\text { Without } \\
\text { Relay }\end{array}$ & 790 & 735 & 565 & 780 \\
\hline
\end{tabular}

where $e$ is the base of the natural logarithm $(e=2.71828 \ldots), r$ is the number of occurrences of an event - the probability of which is given by the function, $\lambda$ is a positive real number, equal to the expected number of occurrences that occur during the given interval. Thus, Poisson distribution is an unprejudiced and unbiased function whose memory less property can be effectively used to determine the sleep mode nodes so as to develop a practical condition in the simulation. We have fixed the occurrence $r$ of the Poisson distribution to be the number of nodes present in the system with $\mathrm{r}=\mathrm{i}$ representing the $(i+1)^{\text {th }}$ identifiable node in the network and we have varied $\lambda$ from 0.8 to 1.6 (taking the value $\lambda$ in this range gives an output in linear matrix form in which the number of sleep mode nodes in the network approaches that of a practical scenario. Too less or more a value of $\lambda$ makes the number of sleep mode nodes undesirably low or high thus violating a practical approach) for our simulation purpose. The above input gave a linear matrix of distribution $(1 \times \mathrm{r})$ with elements having integer value. Output elements with zero output are treated as sleep nodes and automatically satisfy for contending as a relay node for communicating between two nodes. The number of nodes in the topology was taken to be 25 and deployed according to the strategy discussed in 3.2. The power calculations were done according to [17]. The initial energy of each node was set to be 500 mili Joules and the packet length of each communication was set to 100 bits. The node density (the number of nodes in the network divided the area in which they are deployed) was varied from 0.04 to 0.2 by varying the dimensions of the field on which the node was deployed

Moreover, such a range of node density is totally practicable as decreasing the node density creates hole in the network (there may be nodes which has no node in its neighbor to transfer its data) and increasing the number of node automatically decreases the network lifetime. Thus, our approximation discussed in Lemma 1 is satisfied. Four routing algorithms (which use the Euclidean distance between two nodes as one of the parameter for controlling the data transfer) were simulated in this environment, which include PEGASIS (Lindsey and Raghavendra, 2002) [6], SHORT [5], LBEERA [11] and HDS [12] using the algorithm stated in section 3.3. The Poisson distribution function is used to determine which nodes will act as relay node.

The variation of network lifetime with node density with and without the discussed mathematical model is shown in Figure 4, 5, 6 for all four algorithms -SHORT, PEGASIS, LBEERA and HDS respectively for $=0.8,=1.2$ and $=1.6$. The figures depict the considerable improvement of network lifetime using the mathematical model. In table 3, we have tabulated the simulation results of Network Lifetime for different values of. We have compared the Network lifetime results of different data gathering algorithm in the context of with relay and without relay. Here we have varied the value of from 0.8 to 1.6 .

\section{CONCLUSIONS}

Routing in sensor networks is a new area of research, with a limited, but rapidly growing set of research results. In this paper, we have formulated a relay model which utilizes the inactive nodes for adaptive routing. The possibility of nodes to act as a relay is determined by Poisson distribution and the value of lambda. The performance is compared for different data gathering schemes using our relay scheme. From our analysis, we can firmly conclude that our adaptive routing shows notable enhancement of Network Lifetime. This is clear from the result tables and graphs. Our further work will be focused toward formation of a new routing algorithm that will be much more suitable for relay technique.

\section{REFERENCE}

[1] Akyildiz I.F., Su W., Sankarasubramaniam Y., Cayirci E. (2002). Wireless sensor networks: a survey, Computer Networks, Vol. 38, No. 4, pp. 393-422.

[2] Katz R.H., Kahn J.M., Pister K.S.J. (1999). Mobile networking for smart dust, Proceedings of the 5th Annual ACM/IEEE International Conference on Mobile Computing and Networking (MobiCom_99), Seattle, US.

[3] Min R., Bhardwaj M., Cho S.H., Shih E., Sinha A., Wang A., Chandrakasan A. (2001). Low power wireless sensor networks, Proceedings of International Conference on VLSI Design, Bangalore, India.

[4] Rabaey J.M., Ammer M.J., Da Silva J.L., Jr Patel D., Roundy S. (2000). PicoRadio supports ad hoc ultralow power wireless networking, IEEE Computer, Vol. 33, No. 7, pp. 42-48.

[5] Yang Y., Wu H.H., Chen H.H. (2006). Short: shortest hop routing tree for wireless sensor networks, IEEE ICC 2006 Proceedings.

[6] Lindsey S., Raghavendra C.S. (2002). Pegasis: power efficient gathering in sensor information systems, Proceedings of IEEE Aerospace Conference 2002, Big Sky, Montana, pp. 1125-1130.

[7] Seetharam A., Bhattacharyya A., Naskar M.K., Mukherjee A. (2008). Estimation of node density energy efficient deployment scheme in wireless sensor network, Bangalore, India. 
[8] Ye W., Heidemann J., Estrin D. (2002). An energyefficient MAC protocol for wireless sensor networks, Proceedings of the 21st IEEE INFOCOM.

[9] Wang F., Wang D., Liu J. (2011). Traffic-aware relay node deployment maximizing lifetime for data collection wireless sensor networks, IEEE Transaction on Parallel and Distributed System, Vol. 22, No. 8, pp. 1415-1423.

[10] Heinzelman W., Chandrakasan A., Balakrishnan H. (2000). Energy-efficient communication protocol for wireless microsensor networks, Proc. Hawaii International Conference on System Sciences (HICSS33).

[11] Yu Y., Wei G. (2007). Energy aware routing algorithm based on layered chain in wireless sensor network, Wireless Communications, Networking and Mobile Computing, pp. 2701-2704.

[12] Chakraborty A., Mitra S.K., Naskar M.K. (2010). An efficient hybrid data gathering scheme in wireless sensor networks, ICDCIT 2010, LNCS 5966, pp. 98103.

[13] Heinzelman W., Kulik J., Balakrishnan H. (1999). Adaptive protocols for information dissemination in wireless sensor networks, Proc. 5th ACM/IEEE Mobicom Conference (MobiCom '99), Seattle, WA, pp. 174-85.
[14] Kulik J., Heinzelman W.R., Balakrishnan H. (2002). Negotiation-based protocols for disseminating information in wireless sensor networks, Wireless Networks, Vol. 8, pp. 169-185.

[15] Manjeshwar A., Agrawal D.P. (2001). TEEN: a protocol for enhanced efficiency in wireless sensor networks, Proceedings of the 1st International Workshop on Parallel and Distributed Computing Issues in Wireless Networks and Mobile Computing, San Francisco, CA.

[16] Manjeshwar A., Agarwal D.P. (2002). Apteen: a hybrid protocol for efficient routing and comprehensive information retrieval in wireless sensor networks, Parallel and Distributed Processing Symposium, Proceedings International, IPDPS 2002, pp. 195-202.

[17] Mitra S.K., Banerjee J., Chakraborty A., Naskar M.K. (2011). Data gathering in wireless sensor network using realistic power control, ICCCS 2011, Rourkela, India. 